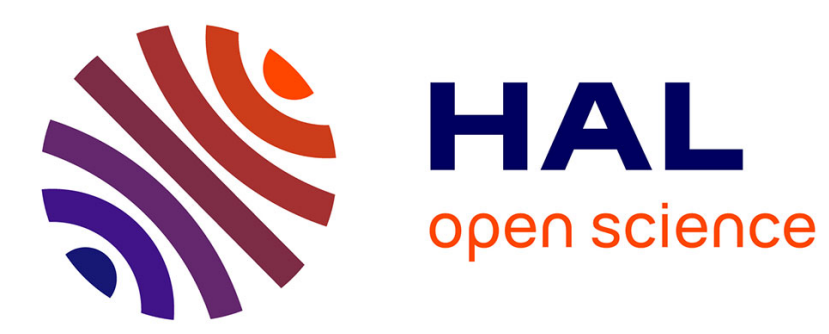

\title{
Valeurs et pratiques de GRH dans l'économie sociale
}

Christophe Everaere

\section{To cite this version:}

Christophe Everaere. Valeurs et pratiques de GRH dans l'économie sociale. Revue Française de Gestion, 2011, 37 (217), pp.20. halshs-00660808

\section{HAL Id: halshs-00660808 https://shs.hal.science/halshs-00660808}

Submitted on 17 Jan 2012

HAL is a multi-disciplinary open access archive for the deposit and dissemination of scientific research documents, whether they are published or not. The documents may come from teaching and research institutions in France or abroad, or from public or private research centers.
L'archive ouverte pluridisciplinaire HAL, est destinée au dépôt et à la diffusion de documents scientifiques de niveau recherche, publiés ou non, émanant des établissements d'enseignement et de recherche français ou étrangers, des laboratoires publics ou privés. 


\title{
Valeurs et pratiques de GRH dans l'économie sociale ${ }^{1}$
}

\author{
Christophe EVERAERE \\ Professeur des Universités \\ IAE - Université Jean Moulin Lyon 3 \\ 6 cours Albert Thomas \\ 69355 Lyon Cedex 08 \\ Tel. (33) 0478787633 \\ Fax. (33) 0478787422 \\ e-mail : christophe.everaere@univ-lyon3.fr
}

\begin{abstract}
Résumé
Une étude menée sur les pratiques de GRH dans les structures de l'économie sociale et solidaire (ESS) en Rhône-Alpes a permis de mettre en évidence la grande hétérogénéité de ce secteur d'activité et ses principes particuliers de fonctionnement. Un salarié sur dix est employé dans ce secteur qui donne à «l'humain » une place en principe privilégiée. Nous avons cherché à cerner les particularités de ce secteur en faisant exprimer par des responsables de ces structures ce qu'ils considèrent être les valeurs spécifiques de l'ESS, et comment ces valeurs énoncées impactent les pratiques de gestion des ressources humaines.
\end{abstract}

\section{Summary:}

A study on HRM practices in social and solidarity economy (SSE) in the Rhone-Alpes has highlighted the big heterogeneity of this sector and its originality. $10 \%$ of employees work in this sector in which people are supposed to be the main resource. We sought to identify the specificities of this sector by asking managers in charge of these structures what they consider to be the values of SSE, and how these values impact on the practices of human resources management.

\footnotetext{
1 Paru dans la Revue Française de Gestion, ${ }^{\circ}$ 217, octobre 2011
} 


\section{L’ESS : un secteur disparate et antagoniste de l'économie classique}

La première chose qui frappe tout observateur de l'ESS est la grande hétérogénéité de nature, de taille et de vocation des structures : on y trouve des clubs sportifs, des coopératives agricoles, des mutuelles d'assurance, des entreprises d'insertion, des congrégations religieuses, etc. Il est d'ailleurs fréquent de les définir par défaut : ni privé marchand, ni administration publique. C'est pourquoi, on les regroupe parfois sous l'intitulé «tiers secteur » (Archambaud, 1996 ; Demoustier, 2006).

Dans son essence même, on insiste souvent sur le caractère alternatif de l'économie sociale : «L'économie sociale est une utopie dans le sens où elle constitue un projet imaginaire de société alternative selon les termes de Henri Desroche (...). L'économie sociale s'est d'abord pensée comme un mouvement alternatif. Des associations ouvrières de production jusqu'à la grande utopie de la république coopérative de Charles Gide, l'économie sociale ambitionne de résister à l'essor du capitalisme, de le dépasser et de le faire disparaître » (Draperi, 2003).

Cette définition «par défaut» ou par opposition de l'ESS semble confirmée par des antagonismes forts avec l'économie classique en termes de localisation géographique. En effet, l'ESS est peu développée sur les territoires où l'économie classique est bien implantée et connaît un certain dynamisme à la fois économique et démographique. De même, un poids important de l'ESS va souvent de pair avec un taux de chômage élevé, c'est-à-dire des zones plutôt rurales qui connaissent des difficultés ou une faible implantation d'entreprises classiques (Diloisi et Dupré, 2007). Un peu comme si l'ESS remplissait un vide et que les deux mondes ne se mélangent pas, voire obéissent à des logiques antagonistes : quand l'un progresse (économie classique), l'autre régresse (ESS); et vice-versa. Toutefois, ce phénomène d'antagonisme ne s'applique pas aux banques coopératives ni aux assurances mutualistes qui sont bien implantées dans le tissu économique et social classique.

$\mathrm{Au}$ delà de cette grande hétérogénéité, les structures de l'ESS partagent quelques principes communs (définis dans une charte commune de l'économie sociale énoncée en 1980) :

Ce sont des sociétés de personnes (adhérents ou sociétaires), et non de capitaux.

Leur gouvernance est démocratique: les décisions sont prises en assemblée générale (les membres de l'AG étant élus par les adhérents) où chacun dispose 
d'une voix (égalitaire) contrairement aux sociétés de capitaux où les droits de vote sont proportionnels aux parts du capital détenu.

- L'appropriation privative du profit n'est pas le but de ces structures créées davantage dans un objectif collectif, partagé et durable ${ }^{1}$.

Trois principales familles composent l'économie sociale et solidaire : les associations, les coopératives et les mutuelles.

Les associations (régies par la loi dite de 1901) représentent $80 \%$ des établissements de l'ESS en Rhône-Alpes. Les activités de ces associations sont elles-mêmes très disparates et concernent notamment l'action sociale (crèches, garderies d'enfants, structures d'aide à domicile, accueil des adultes et enfants handicapés, etc.), l'éducation (établissements privés d'enseignement, les centres de formation des apprentis, etc.), la santé (centres hospitaliers, par exemple), les sports, culture \& loisirs (les clubs sportifs, l'Opéra de Lyon, la Maison de la Culture de Grenoble, etc.), l'hébergement, accueil \& restauration (comités de vacances, fédérations des œuvres laïques, centres aérés, etc.), les services aux entreprises (Pays de Gex Insertion Entreprises, l'APAVE, par exemple), des associations de personnes (couvents, association d'aide des prêtres missionnaires, etc.), des associations diverses (les MJC, les fondations, etc.).

Avec $16 \%$ des établissements de l'ESS, les coopératives sont des regroupements de consommateurs, des coopératives scolaires, de salariés (les SCOP) ou d'entrepreneurs (les coopératives agricoles ou de commerçants détaillants comme Système U) qui poursuivent des buts communs. On trouve donc des coopératives agricoles, des coopératives de production et des coopératives de crédit (les caisses de Crédit Agricole Mutuel, le Crédit Coopératif, les sociétés coopératives de Banque Populaire, etc.). Mais près de la moitié des coopératives sont regroupées dans la rubrique «autres »: BTP, services intellectuels et culturels, les Messageries Lyonnaises de Presse (MLP), etc.

Enfin, les mutuelles d'assurance et de prévoyance $(2,6 \%$ des établissements de l'ESS en Rhône-Alpes) appliquent le principe de solidarité en répartissant les coûts de la prévention et

1 Pour clarifier ce dernier point : «quand une association ou une coopérative dégage un surplus économique important, elle préfère généralement l'investir dans l'embauche d'un salarié supplémentaire, ou dans 
la répartition des risques. Certaines sont bien connues comme la MAIF, la MACIF, la MGEN, la MSA, etc.

La région Rhône-Alpes se situe dans la moyenne nationale en termes de représentation de l'ESS par rapport à l'ensemble des établissements dénombrés par l'INSEE (répertoire Sirène) : $10 \%$ des établissements en Rhône-Alpes, avec des écarts qui varient du simple au double : 6,5 \% pour la Corse, contre $13 \%$ pour les pays de la Loire (CNESS, 2008). A l'échelle nationale, l'ESS (mutuelles, coopératives, associations et fondations) représente 800000 entreprises qui emploient 1,8 million de salariés. A l'échelle européenne, l'ESS compte 150 millions de membres et 10 millions de salariés ${ }^{1}$.

Si l'on mesure le poids de l'ESS, non plus en en termes de nombre d'établissements mais en termes d'effectif salarié, ce sont $\mathbf{1 0} \%$ des salariés de la région Rhône-Alpes qui travaillent dans l'ESS (proportion identique à la moyenne nationale (soit 2,1 millions de salariés en tout) et européenne). Corrélativement au nombre d'établissement, les associations concentrent $78 \%$ des emplois de l'ESS, contre $14 \%$ pour les coopératives et $6 \%$ pour les mutuelles (à l'échelle nationale ; source : CNESS, 2008).

\section{L'ESS : un projet ambitieux et prometteur pour la GRH}

De par ses fondements, l'économie sociale et solidaire constitue un projet très ambitieux et prometteur pour les ressources humaines.

En effet, «l'économie sociale est un mouvement qui vise à impliquer des individus dans des formes démocratiques d'organisation où les préoccupations sociétales (sociales, civiques, environnementales et/ou équitables) priment sur l'économie. Ces personnes s'impliquent dans le même temps comme membres et comme salariés ou usagers ; elles gèrent l'entreprise ensemble sur une base égalitaire ». (Jeantet, 2006). Appliquée à la GRH, la recherche d'un principe d'égalité questionne en particulier les pratiques de rémunération. Les écarts de salaire seraient-ils moins élevés en ESS que dans les entreprises classiques?

\footnotetext{
l'amélioration des conditions de travail (matérielles ou non) de son personnel, alors qu'une entreprise privée capitaliste l'utiliserait pour mieux rétribuer ses actionnaires (Davister, 2006, p. 13)

${ }^{1}$ source : La lettre du GNC (Groupement National de la Coopération), nº 348, juin 2007, page 9.
} 
«Les définitions de l'économie sociale soulignent le principe de primauté du facteur travail dans la répartition des revenus. Ainsi, l'idéologie de l'économie sociale place les travailleurs (salariés et bénévoles) au cœur même du processus de production (...). Alors que le secteur capitaliste privilégie la rémunération du capital financier (rétribution des investisseurs principalement) et le développement du capital technique (nouveaux équipements), les organisations d'économie sociale privilégient le développement du capital humain. Par conséquent, elles sont amenées à développer des pratiques de GRH qui traduisent concrètement cette valeur de primauté du travail » (Davister, 2006, p. 13). L'importance et la primauté du facteur humain sont ici clairement exprimées. Quels impacts concrets ces principes peuvent-ils avoir sur les pratiques de GRH ?

C'est toute l'idéologie même de l'ESS qui fait de l'amélioration du sort de l'humain le but même des activités économiques, ceci en rupture avec le système capitaliste classique : «Apparue au milieu du 19è siècle, la notion d'économie sociale puise ses racines dans différentes écoles de pensée :

- un courant socialiste associationniste qui vise à émanciper les travailleurs d'un pouvoir capitaliste dominant,

- $\quad$ une tendance sociale-chrétienne fondée sur la doctrine sociale de l'Eglise et qui défend l'idée de solidarité envers les plus défavorisés,

- $\quad$ une tradition libérale qui encourage les initiatives d'entraide des ouvriers,

- et une école solidariste qui prône le coopératisme et soutient l'apparition d'organisations fonctionnant selon les principes démocratiques » (Persais, 2006, p. 21)

Comment dans ces conditions appréhender la notion de gestion des ressources humaines dans les structures de l'ESS ? Les spécialistes du sujet ont déjà noté un point saillant sur le concept même de «ressources » humaines : «Le concept de GRH est souvent mal perçu par les dirigeants d'économie sociale parce qu'il est souvent associé dans leur esprit à une conception capitaliste des travailleurs comme constituant une « ressource » à exploiter au même titre que les autres ressources (financières, matérielles, énergétiques, etc.) de l'entreprise. Pour bon nombre de responsables d'organisations de l'économie sociale, ce concept fait partie d'un langage purement économique dont ils se méfient car celui-ci ne reflète pas la manière dont l'économie sociale considère ses «travailleurs » (individus qui réalisent une activité économique pour accomplir une mission sociale) et ne prend pas en 
compte les caractéristiques des organisations d'économie sociale (leur finalité sociale et les valeurs qui guident leur fonctionnement interne) (Davister, 2006, p. 9).

D'autres points sont évoqués : «les organisations d'économie sociale semblent gérer leurs ressources humaines de manière plus intuitive et informelle » (ibid. p. 3). Ce point rejoint des analyses menées par ailleurs sur la GRH en TPE (très petites entreprises : globalement moins de dix personnes): Les dirigeants de TPE adoptent en effet un schéma de décision plutôt réactif, opportuniste ou spontané. Ce schéma de décision se retrouve dans les décisions relatives au personnel et rend la GRH plutôt opportuniste, intuitive, voire improvisée (notamment en matière de recrutement) plus que réfléchie, structurée (bureaucratique), anticipée (comme c'est davantage le cas en grande entreprise). De la même façon, les dirigeants de TPE utilisent davantage le terme de «personnel» que celui de «ressources humaines » qui leur paraît trop anonyme ou distant (Torrès et Delmar, 2006).

Cette correspondance entre la GRH en ESS et la GRH en TPE est logique puisque l'ESS est marquée par la très petite taille de ses établissements ${ }^{1}: 61 \%$ des établissements sont des structures sans salarié. La part des établissements qui comptent plus de 50 salariés est seulement de $3 \%$ dans le cas des associations et des coopératives, et $7 \%$ dans le cas des mutuelles (les mutuelles concentrent les établissements dotés des plus grands effectifs). $80 \%$ des associations (qui dominent le secteur de l'ESS) emploient moins de 10 salariés et plus de la moitié des établissements employeurs dans le secteur associatif de Rhône-Alpes n'ont qu'un ou deux salariés seulement. Plus de la moitié des coopératives comptent entre 3 et 9 salariés en Rhône-Alpes ${ }^{2}$.

Pourtant, l'ESS comporte aussi de très grandes structures : la Croix Rouge (française), par exemple, compte 16300 salariés, 50000 bénévoles et gère 900 millions de budget. La branche allemande de la Croix Rouge est encore plus grande avec ses 72000 salariés ${ }^{1}$. L'association des Paralysés de Frances (APF) est classée dans le top 10 des entreprises de services en France, avec ses 15000 salariés, 420 établissements, et ses 500 millions d'Euros de chiffre d'affaires. Ces contrastes de taille rendent difficile tout exercice de généralisation

\footnotetext{
${ }^{1}$ Dans l'étude de l'INSEE (2003) consacrée à l'ESS en Rhône-Alpes, un établissement correspond à une adresse : une même entreprise ou association peut donc compter plusieurs établissements.

${ }^{2}$ A l'échelle nationale, les effectifs salariés moyens par établissement varient dans une fourchette de 1 à 6 , avec une moyenne de 10 salariés pour les associations, 13 pour le coopératives, 17 pour les mutuelles, soit une moyenne de 11 salariés par employeur pour l'ensemble de l'ESS (CNESS, 2008).
} 
des pratiques de GRH en ESS. Nous y revenons ensuite pour ajouter à ces écarts de taille, d'autres écarts relatifs à la nature même de l'activité menées en ESS.

C. Davister (ibid. p. 17) note également une certaine réticence à utiliser en ESS des outils de gestion importés du secteur privé classique, notamment ceux qui concernent les outils d'évaluation et de contrôle. «Le fait que ces organisations aient des difficultés à définir clairement leur mission et les besoins de leurs clients rend l'évaluation de leur performance délicate ».

\section{Des activités très hétérogènes en ESS}

En plus des contrastes de taille, la nature même de l'activité des structures en ESS est très disparate. $65 \%$ des emplois sont occupés par des femmes en ESS contre $41 \%$ dans l'ensemble du secteur économique de Rhône-Alpes, hors secteurs ESS. Ceci peut s'expliquer par l'importance de la production de services plutôt que de biens matériels dans l'ESS en général. De plus, les services en question recouvrent souvent des activités dans lesquelles les femmes jouent traditionnellement une part plus importante que les hommes : santé, services sociaux, services aux personnes, éducation, services domestiques, etc.

Cette plus forte proportion de femmes explique à son tour une caractéristique forte de l'emploi en ESS, qui est celle de la pratique importante du travail à temps partiel $(3 / 10$ des emplois sont à temps partiel en ESS ${ }^{2}$, soit quasiment le double de la moyenne, tous secteurs confondus).

\footnotetext{
${ }^{1}$ M. Bellan, « La Croix Rouge fait des RH un de ses leviers de transformation », Les Echos, 3 avril 2007.

2 Trois explications possibles à ce phénomène :
}

- Une explication démographique en rapport avec la présence plus importante des femmes dans l'ESS, lesquelles sont traditionnellement plus attirées par cette forme d'emploi que les hommes ( $83 \%$ des salariés à temps partiel sont des femmes (Ulrich et Zilbermann, 2007).

- Une deuxième explication possible réside dans les valeurs supposées particulières de l'ESS : "On reconnaît la volonté de l'économie sociale de respecter un équilibre entre vie professionnelle et vie privée, alors qu'une entreprise classique cherche avant tout à gérer de manière performante ses ressources humaines. En ce sens, on pourrait dire que la GRH en économie sociale est avant tout une gestion de l'humain (Davister, 2006, p. 29).

- Une troisième explication relève de l'effet taille. En effet, structurellement, les TPE recourent davantage à des formes d'emploi à durée limitée, au temps partiel ou à des contrats aidés par l'Etat que les entreprises de 10 salariés ou plus. En juin 2007, $24 \%$ des salariés des TPE étaient employés à temps partiel (ce taux était de $33 \%$ en 2002), contre $16 \%$ dans les entreprises de 10 salariés et plus (Berry et Dubois, 2008).

On retrouve dans ces hypothèses explicatives le tiraillement entre plusieurs paramètres distincts : le paramètre démographique (population plus féminine), le paramètre «philosophique » des valeurs supposées plus humaines en ESS, le paramètre «structurel » de la taille des établissements. 
Cependant, on repère des contrastes au sein de l'ESS : le taux de féminisation de l'emploi est supérieur dans les mutuelles : $75 \%$ des emplois y sont occupés par des femmes contre $67 \%$ dans les associations. A l'inverse, la population est majoritairement masculine (59\%) dans les coopératives de production dont les activités comportent une dimension plus industrielle, et donc plus masculine.

Tout comme l'ensemble de l'ESS dont il est le principal constituant (80\% des établissements et des effectifs), le secteur associatif ${ }^{1}$ est lui-même un groupe très hétérogène dans lequel le social (aide à domicile des personnes âgées, travail protégé, aide aux enfants infirmes ou inadaptés, etc.) représente plus du tiers des emplois de l'ESS en Rhône-Alpes.

Si le secteur de la santé ne représente que $7 \%$ des emplois du secteur associatif, il en vaut le double en termes de masse salariale. On peut y voir la conséquence d'un niveau de qualification, et donc de rémunération, plus élevé dans le secteur de la santé par rapport à d'autres secteurs associatifs moins exigeants en niveau de formation ou d'études.

\section{Le niveau de qualification varie donc beaucoup selon les secteurs d'activité au sein du}

monde associatif : dans l'éducation, la santé, la culture (sport et loisirs), les cadres et professions intermédiaires dépassent la moitié des effectifs; tandis qu'ils ne représentent qu'entre le quart et le tiers des effectifs dans le social, les services d'accueil, hébergement, restauration et les associations de services aux entreprises (Insee, 2003, p. 33).

Les problématiques de management, autour de la question-clé des compétences (et ce qui s’y rattache : des contrats de travail plus stables, du temps de travail moins partiel, des éventuelles difficultés de recrutement compte tenu de la rareté de certaines compétences critiques, des niveaux de rémunération plus élevés, etc.), varient donc beaucoup selon les associations et la nature de leurs activités : simples ou complexes (ce paramètre pouvant être mesuré par le niveau de qualification du personnel requis dans les unités opérationnelles).

\footnotetext{
${ }^{1}$ Le nombre d'associations vivantes et actives est estimé à 1,1 million (à partir d'une enquête menée en 2005). 172000 associations recourent à l'emploi salarié (1,9 million de personnes concernées). Par déduction, 928000 associations ne s'appuient que sur une catégorie bien particulière de ressources que sont les bénévoles pour mettre en place leur projet (Tchernonog, 2008). Cette question des bénévoles constitue un autre sujet particulier à l'ESS qui mériterait un développement spécifique. Cependant, pour confirmer les contrastes en ESS, si un grand nombre de petites associations (notamment dans le secteur sportif et culturel), fonctionnent grâce au travail bénévole, cette question ne concerne ni les coopératives, ni les mutuelles.
} 
Par exemple, le secteur de l'aide à domicile des personnes âgées occupe 99 \% de femmes, les emplois y sont marqués par une très grande précarité : prédominance du travail à temps partiel sur des durées très faibles (dix heures par semaine), les qualifications y sont très faiblement reconnues et les rémunérations, très basses, sont souvent proches des seuils de pauvreté. Alors que les services hospitaliers, à domicile, pour ces mêmes personnes âgées obéissent à des logiques complètement opposées : emplois stables, diplômes exigés, temps partiel choisis, rémunérations plus élevées (Clergeau et Dussuet, 2004). D'où l'énorme difficulté à énoncer des généralités en matière de management dans les associations en particulier, et dans l'ESS en général.

Après avoir apporté quelques éléments factuels concernant les structures de l'ESS, en insistant sur les principaux éléments relevant des origines, des fondements, du travail et de l'emploi, nous allons maintenant présenter les résultats d'une étude que nous avons menée sous forme d'enquêtes auprès de responsables d'associations et de coopératives en région Rhône-Alpes.

\section{Résultats d'une enquête sur les liens entre les valeurs et les pratiques de GRH en ESS}

L'un des objectifs de cette enquête a été de cerner les liens entre les valeurs supposées particulières de l'ESS et les pratiques de gestion des ressources humaines. Cet objectif étant lui-même inscrit dans l'optique de constituer un pôle de compétences GRH pour les entreprises de l'ESS en Rhône-Alpes, à l'initiative du Collège Coopératif Rhône-Alpes.

Par souci de concision, nous ne présenterons pas l'ensemble du protocole de cette étude. Le questionnaire, envoyé par courrier électronique aux responsables des structures adhérentes de l'URIOPSS ${ }^{1}$ de l'URSCOP $^{2}$, comprenait huit questions ouvertes sur les enjeux et pratiques de GRH en ESS, et treize questions factuelles destinées à préciser les caractéristiques de la structure concernée.

\footnotetext{
${ }^{1}$ Union Régionale Interfédérale des Organismes Privés non lucratifs Sanitaires et Sociaux de Rhône-Alpes

${ }^{2}$ Union Régionale des Sociétés Coopératives de Production Rhône-Alpes
} 
Parmi les résultats qui nous intéressent directement figurent les réponses aux deux questions qui ont été formulées de la façon suivante :

Question 3: Quelles sont pour vous les valeurs caractéristiques des structures de l'Economie Sociale et Solidaire? (Exprimez ce qui vous vient spontanément à l'esprit).

\section{Question 4 : En quoi les valeurs de l'ESS impactent vos pratiques de GRH ?}

Pouvez-vous donner des illustrations, par exemple, dans les domaines du recrutementintégration, de la rémunération, de la formation, de l'organisation du travail, de la gestion et évaluation des compétences, des modalités de rupture du contrat de travail, etc.

\subsection{Composition de l'échantillon étudié}

Le Collège Coopératif Rhône-Alpes a procédé à l'envoi du questionnaire à près de 550 adresses électroniques, sur la région Rhône-Alpes, extraites des fichiers des adhérents des structures partenaires de l'étude : l'URIOPSS et l'URSCOP.

Au total, nous avons reçu 25 réponses exploitables au cours du mois de novembre 2008. Compte tenu du faible nombre des retours, en aucun cas, nous ne pouvons prétendre à une quelconque représentativité des réponses fournies par rapport à l'ensemble des structures de l'ESS en Rhône-Alpes. Le caractère exploratoire de notre étude, ainsi que le degré de sensibilité et d'intérêt très inégal des structures à notre enquête empêchent la moindre extrapolation statistique. Cependant, il nous semble que les réponses, même limitées en nombre, fournissent quelques résultats intéressants.

Parmi les 25 répondants, figurent 16 associations, 7 coopératives, 1 fondation et 1 mutuelle. Leurs caractéristiques principales sont mentionnées à la suite des citations analysées dans la suite du document.

Si notre échantillon est limité en volume, il confirme cependant la forte représentativité des associations, ainsi que la grande hétérogénéité à la fois des activités caractéristiques de l'ESS (un bureau d'étude dans le bâtiment côtoie des structures de soin, ainsi qu'un magasin de 
produits biologiques et un sous-traitant industriel) et de la taille des structures (des structures de 400 personnes côtoient de toutes petites unités de moins de 5 personnes).

\subsection{Les valeurs caractéristiques de l'ESS (exprimées par les répondants)}

Toutes les structures ont répondu à cette question. Les propos restitués ici sont tels qu'ils ont été formulés et typographiés (en dehors des fautes de frappe ou de syntaxe corrigés par nousmême) par les répondants dans le courrier électronique de réponse au questionnaire. Ce qui explique que certains propos copiés-collés apparaissent en majuscule, car c'est ainsi que les répondants nous ont transmis leur réponse.

- «accueil, écoute, comprendre » (citation de LB : maison d'enfants à caractère social, 70 salariés en ETP $^{1}$, dont $80 \%$ de femmes).

- « respect de l'autre, partage, sens des responsabilités » (citation de TPC : Entreprise Adaptée dans l'insertion professionnelle des personnes handicapées, 180 personnes en ETP, dont $80 \%$ de personnes handicapées).

- « solidarité, entraide, responsabilité, partage, non-discrimination » (citation de ESE : $\mathrm{ESAT}^{1}$ en sous-traitance industrielle, 394 salariés, pas de bénévole).

- «SOLIDARITE, réduction des inégalités, acceptation de la vulnérabilité des personnes, proximité, lien social» (citation de AA : association d'accueil de personnes alcooliques et en situation d'addiction, 7 salariés en ETP +3 bénévoles).

- «priorité au service des personnes face aux contraintes et enjeux économiques » (citation de PV : association de santé spécialisée dans la déficience auditive : 56 salariés dont beaucoup à temps partiel, $8 \%$ de l'effectif est composé de personnes handicapées).

- «engagement, qualité de service, individualisation de la prestation » (citation de AS : fondation spécialisée dans la lutte contre l'exclusion, 60 personnes en ETP +60 personnes à mi-temps en contrats aidés (chantiers d'insertion) + des bénévoles).

${ }^{1}$ ETP : Equivalent Temps Plein 
- «la responsabilité individuelle au service de la solidarité et l'échange (citation de SO: coopérative d'activité et d'emploi, 28 salariés en ETP pour un effectif total de 76 personnes).

- «écoute, échange, partage de valeurs communes » (citation de AGI : association spécialisée dans l'accueil d'enfants et d'adultes handicapés mentaux, 250 salariés en ETP +2 bénévoles).

On peut noter ici que les valeurs exprimées s'appliquent autant au public concerné par l'activité des associations : «accepter la vulnérabilité des personnes » pour AA qui accueille des personnes alcooliques ; «qualité de service, individualisation de la prestation » pour AS qui est une fondation spécialisée dans la lutte contre l'exclusion; qu'aux collaborateurs internes : "rapport au travail qui ne se limite pas au salaire, et qui (je veux le croire) permet à chacun de trouver une motivation personnelle dans son action » (citation de IJG, accueil de personnes âgées); "Permettre à chaque salarié de s'épanouir dans ses fonctions, d'évoluer dans ses fonctions, de se former pour évoluer dans ses compétences et avoir un salaire en adéquation avec sa fonction» (citation de TC, coopérative d'activité et d'emploi), «solidarité, entraide, responsabilité, partage, non-discrimination » dans le cas de ESE (un ESAT en soustraitance industrielle).

Cette attention aux valeurs qui s'adressent au public (extérieur) peut s'expliquer par la nature tertiaire de l'activité de ces associations. A l'inverse, il n'est pas surprenant qu'un ESAT qui travaille en sous-traitance industrielle privilégie l'expression de valeur à l'attention des salariés (en l'occurrence handicapés), puisque le client est unique et parfaitement connu.

Ce double regard (externe et interne) est explicitement exprimé par l'une des associations : «Sociale et solidaire au sens large : c'est à dire entreprise sociale autant pour les salariés permanents que pour les personnes accueillies » (citation de IME : Institut Médico Educatif, spécialisé dans l'accueil d'enfants et adultes sourds avec troubles associés, 200 salariés, dont $10 \%$ de personnes handicapées).

Le regard est plus large encore dans le cas de LTD (magasin de produits biologiques en SCOP) puisqu'il est question de développement durable et des nombreuses parties prenantes : «Respect des salariés, des clients, des producteurs (pour ceux qui travaillent comme nous avec des petites structures), de l'environnement... Sensibilité à l'environnement dans lequel

\footnotetext{
${ }^{1}$ ESAT (ex-CAT) : Etablissement et Structures d'Aide par le Travail
} 
ces structures évoluent. Solidarité entre les structures. Entraide. Non pratique du principe de la terre brûlée, inscription dans une démarche Durable».

D'autres réponses méritent également d'être restituées :

«Les structures de l'Economie Sociale et Solidaire permettent, à mon avis, de faciliter les projets, d'importances différentes, humanitaires et solidaires. Ceci devrait permettre à des jeunes ou moins jeunes de plus en plus nombreux de s'impliquer auprès de personnes en difficulté » (citation de AV : association dans le domaine de l'hébergement d'adultes en situation de handicap mental, 25 salariés +13 volontaires +2 bénévoles).

«Non lucrativité - solidarité - Démarche participative et innovante pour une qualité de services et de relations - Responsabilité et réalisme nécessaire en gestion économique » (citation de BT : structure regroupant 4 associations de taille variable (20 à 120 salariés) dans des domaines divers : soins à domicile, insertion par l'économique, accueil des populations étrangères, formation en alternance pour adultes).

«Le respect des droits des personnes, éthique et déontologie, engagement professionnel, démarche d'amélioration continue et qualité des prestations » (citation de CAP: association d'accueil d'adultes handicapés, 100 salariés +20 bénévoles).

«Il s'agit de placer la personne au centre de nos intérêts : respect de la dignité de la personne. L'éthique et la philosophie associative : à but non lucratif, bénévoles, adhésion aux fondamentaux de l'association. Intégrité de la personne » (citation de $\mathrm{AD}$ : association spécialisée dans l'accueil de personnes handicapées mentales, 241 salariés en ETP).

L'un des répondants (responsable d'un centre de santé, 45 salariés) a tenu à faire un parallèle intéressant entre son expérience actuelle en ESS et son expérience passée en entreprise classique, afin de souligner le contraste de valeurs et de priorité entre les deux mondes :

«Mes réponses sont à mettre en face d'une expérience de 10 ans dans un groupe industriel international

. Implication dans le travail (les nombreuses restructurations dans le secteur industriel ont transformé des personnes impliquées en personnes désabusées. Nous n'avons pas encore connu cela dans le secteur [de l'ESS] (et encore sur les gros pôles hospitaliers?) 
. L'envie de bien faire pour le public passe avant celle de faire plaisir à "l'actionnaire». (Dans le secteur industriel, l'envie de faire plaisir au client passe en second plan par rapport au fait de servir les actionnaires : la crise actuelle n'est qu'une confirmation de plus)

. Travailler sur un secteur géographique palpable : on agit en local, et non pas de manière mondialisée (les patients, clients, usagés... ont des visages, alors que je n'ai que rarement croisé un client lors de mon passage en secteur industriel : ils étaient en Asie, au Canada, en Russie, etc...»

Ces citations nous paraissent très riches et témoignent d'un niveau de réflexion et de recul important sur les pratiques (et leurs sens). De même, le niveau de complexité dans l'expression des valeurs et du sens des actions menées va bien au-delà du classique et simple «profit » ou « retour sur investissement $(\mathrm{ROI}) »$ que l'on trouve davantage dans l'économie classique.

Le phénomène de la proximité («travailler sur un secteur géographique palpable, agir en local, connaître des visages... ») exprimé dans la citation ci-dessous nous semble également important en ce sens que cette proximité induit une relation humaine concrète, solide et durable ${ }^{1}$. A l'inverse, d'une approche virtuelle, globale ou mondialisée, anonyme, distanciée, impersonnelle, réversible ou éphémère que véhicule davantage l'économie classique.

Les SCOP (sociétés coopératives ouvrières de production) insistent davantage sur la gouvernance démocratique :

- «l'accès aux informations relatives à la marche de l'entreprise » (citation de PRO : bureau d'études spécialisé dans le bâtiment, 20 personnes dont 16 ingénieurs et techniciens).

- « solidarité, équité, démocratie participative coopérative» (citation de AFO: organisme de formation continue pour les travailleurs sociaux, 4 salariés permanents +60 intervenants formateurs vacataires).

Insistons cependant sur l'expression de valeurs que nous qualifions de courantes ou banales:

\footnotetext{
1 Cette importance attachée à la proximité, associée à la confiance ou encore au «voisinage » semble bien correspondre à l'ESS: «les entreprises de l'économie sociale considèrent le territoire non comme une opportunité, mais comme la base de leur activité et de leur développement » (Colletis et al., 2004)
} 
- «qualité de services et des relations, responsabilité et réalisme nécessaire en gestion économique » (citation de BT : structure regroupant 4 associations de taille variable (20 à 120 salariés) dans des domaines divers : soins à domicile, insertion par l'économique, accueil des populations étrangères, formation en alternance pour adultes).

- « engagement professionnel, démarche d'amélioration continue et qualité des prestations » (citation de CAP : association d'accueil d'adultes handicapés, 100 salariés +20 bénévoles).

Globalement, les valeurs exprimées, à l'exception des deux dernières citations qui évoquent davantage des préoccupations d'efficacité ou de performance économique classiques, cadrent donc bien avec la place privilégiée accordée à « l'humain » (qu'il soit client ou salarié) dans la finalité et le fonctionnement des structures qui en font partie.

\subsection{Impact des valeurs de l'ESS sur les pratiques de GRH}

Les réponses fournies à cette question traduisent à la fois des problématiques «objectives » (dérivées de l'activité et des spécificités de la structure) et des valeurs « subjectives », dont nous pouvons constater ci-dessus qu'elles comportent nombre de points convergents, notamment la solidarité, l'engagement, les valeurs, le respect de l'autre, etc.

Toutes les structures n'ont pas répondu à cette question, et toutes les rubriques n'ont pas été remplies.

Nous allons classer les réponses en trois catégories :

- les pratiques induites par la spécificité des collaborateurs (volontaires, personnes handicapées) et des structures, en particulier, la question de la gouvernance démocratique inhérente aux coopératives.

- les pratiques que nous qualifions de banales, car identiques à celles que l'on peut trouver dans des entreprises classiques.

- les pratiques où un impact «valeurs ESS » se fait sentir dans les pratiques de GRH.

Parmi les réponses fournies, plusieurs structures insistent les spécificités de leurs collaborateurs. C'est le cas notamment de AV (association dans le domaine de l'hébergement d'adultes en situation de handicap mental, 25 salariés +13 volontaires +2 bénévoles) qui recourt à des 
volontaires ${ }^{1}$ à hauteur d'un tiers des effectifs et qui insiste sur la GRH de cette population particulière :

- recrutement / intégration : Nous avons mis en place tout un système de recrutement et tout un processus d'intégration (stage de découverte, stage d'un mois, ...)

- rémunération : Les volontaires reçoivent tous un pécule

- formation : Nous mettons en place une formation interne de minimum 40h dans l'année, ainsi qu'une formation régionale pour l'ensemble des volontaires de $\mathrm{AV}$.

- organisation du travail : Chaque volontaire est associé à une équipe de 3 à 4 personnes, chargée de l'encadrement et de l'accompagnement d'un groupe de 8 personnes ayant un handicap mental

- gestion \& évaluation des compétences : Pour les volontaires, nous mettons en place une évaluation à la fin de la première semaine et à la fin du mois de stage. Pour les salariés, nous mettons en place tous les 2 ans une évaluation fonctionnelle et tous les 2 ans un entretien de développement afin de vérifier avec le salarié ses attentes et besoins.

- rupture du contrat de travail : Pour les volontaires, nous tentons, tout au long de l'année, de les aider à construire avec eux leurs projets d'avenir.

Dans le cas de l'un des ESAT (ESE : ESAT en sous-traitance industrielle, 394 salariés, pas de bénévole), le répondant a mentionné un taux unique de rémunération pour le personnel (handicapé) en production.

Les SCOP, de leur côté, insistent sur une « gestion coopérative du plan de formation », et sur le recrutement de salariés désireux de devenir co-propriétaires de l'outil de production : «Les conditions objectives (statutaires) impactent les GRH. Le fait que tout salarié peut devenir associé fait tendre à choisir non plus seulement sur des critères « techniques » mais aussi sur une appétence à la co-propriété de l'outil de production et à la finalité sociale de l'entreprise » (citation de CS : coopérative d'emploi et d'activité dont l'activité consiste à aider des personnes à créer leur entreprise, effectif de 7 personnes).

\footnotetext{
${ }_{1}$ Volontaire associatif : La loi relative au volontariat associatif promulguée le 23 mai 2006 a créé un nouveau statut de volontariat et donc un nouveau contrat écrit qui organise une collaboration désintéressée entre l'organisme agréé (une association, une fondation, une union ou une fédération d'association) et la personne volontaire. Le volontariat a pour vocation de devenir le troisième pilier des ressources humaines des associations parallèlement au bénévolat et à l'emploi associatif salarié ; il comble ainsi un manque réel en permettant à un jeune de s'investir pleinement pour une durée déterminée dans un projet d'intérêt général et il constitue également un levier indispensable pour le développement du mouvement associatif dans son ensemble. Source: http://www.jeunesse-sports.gouv.fr/associations 3/volontariat-associatifengagement educatif 238/volontariat-associatif 1178.html
} 
D'autres réponses apparaissent classiques ou banales, dans le sens où elles correspondent à ce qui est décrit et préconisé dans les manuels courants de GRH. Ainsi, en matière de recrutement - intégration, CAP (association d'accueil d'adultes handicapés, 100 salariés +20 bénévoles) mentionne le fait de mettre en place une procédure de recrutement clairement définie avec accompagnement de chaque nouvel embauché dans les mois qui suivent son intégration.

Une autre association insiste sur la priorité accordée au critère du professionnalisme dans le recrutement: «nous devons rechercher des professionnels aptes à travailler avec des populations difficiles, socialement et culturellement, souvent déconnectées des réalités quotidiennes. Nous devons recruter les meilleurs pour offrir à nos usagers la prestation la plus pertinente. Nous ne faisons pas de recrutement «social » (LB : maison d'enfants à caractère social, 70 salariés en ETP, dont $80 \%$ de femmes).

Ce dernier propos tranche nettement avec ce qui est évoqué concernant la GRH en ESS. Dans le cadre d'un recrutement, par exemple, C. Davister évoque le dilemme entre le besoin de compétence pour la structure ou le besoin d'emploi pour la personne : «lors du recrutement, une association doit-elle choisir un comptable expérimenté afin de rationaliser sa gestion ou une personne - moins qualifiée - motivée pour sortir de sa situation de non emploi » (Davister, 2006, p. 20). En l'occurrence, LB a choisi de donner la priorité à la logique organisationnelle.

Autre réponse qualifiée de classique : «En matière d'intégration, nous envisageons de faire une demi journée d'information sur l'histoire et les actions de la congrégation afin de développer une meilleure compréhension du fonctionnement de la structure et de replacer l'action de chacun dans un contexte collectif » (citation de PSP : maison de retraite, 400 salariés dont $85 \%$ de femmes, $5 \%$ de bénévoles).

Au sujet de la rémunération, une SCOP (TPC : Entreprise Adaptée dans l'insertion professionnelle des personnes handicapées, 180 personnes en ETP, dont $80 \%$ de personnes handicapées) mentionne une échelle de salaires de 1 à 7 , ce qui paraît conforme aux écarts de salaire que l'on peut trouver dans les 
entreprises d'une certaine taille ${ }^{1}$. Mais on est loin du principe d'égalité évoqué au début comme fondement de l'ESS.

Dans certaines structures, la formation est présentée comme un levier (classique) de progression des compétences: «c'est un axe fort de la GRH; tant individuelles que collectives pour adapter les compétences requises aux exigences des postes que pour accompagner les transformations des pratiques professionnelles induites par les lois de 2002 \& $2005 »$ (citation de CAP : association d'accueil d'adultes handicapés, 100 salariés +20 bénévoles).

Un répondant dissocie même clairement l'enjeu de la formation de la question des valeurs de l'ESS : «La formation est surtout abordée comme une réponse à des enjeux de professionnalisation, d'évolution et d'adaptation de nos services, de développement de la qualité ou de mise en adéquation avec la réglementation. Les valeurs de l'économie sociale ne sont pas prises en compte » (citation de PSP : maison de retraite, 400 salariés dont $85 \%$ de femmes, $5 \%$ de bénévoles).

En matière de gestion et évaluation des compétences, plusieurs structures mentionnent le classique recours à l'entretien annuel d'évaluation (« entretien annuel pour tous » coopérative TPC : Entreprise Adaptée dans l'insertion professionnelle des personnes handicapées, 180 personnes en ETP, dont $80 \%$ de personnes handicapées), en précisant «pour mesurer l'adéquation entre attente et service rendu » (citation de LB : maison d'enfants à caractère social, 70 salariés en ETP, dont $80 \%$ de femmes). La logique de performance est sous-jacente dans ces propos alors même que les dirigeants de l'ESS manifestent - en principe - une certaine réticence à contrôler les actions des individus engagés dans le projet ${ }^{2}$.

Un répondant prend soin de segmenter la population concernée par la question de la performance : «La gestion des compétences est variable selon l'enjeu du poste de travail. En effet, pour les collaborateurs dont la mission est de s'occuper au quotidien de la personne âgée, l'évaluation des compétences par les managers est à la fois plus rigoureuse et plus

\footnotetext{
${ }^{1}$ Un patron est payé 52700 Euros en moyenne par an (selon l'INSEE). Le salaire d'un patron de PME de 50 personnes est trois fois supérieur à celui d'un ouvrier, selon la CGPME. En revanche, le salaire moyen d'un dirigeant d'une société de plus de 250 salariés est de 188.700 euros. Dans ce cas, l'échelle de salaire passe de 1 à 10. (Le Figaro, 12 février 2009).

${ }_{2}^{2}$ "L'idéologie sur laquelle se fonde l'économie sociale tend à s'opposer à toute forme de pouvoir et donc de contrôle sur les actions des individus engagés dans le projet. Dans un tel contexte, les procédures de gestion
} 
centrée sur l'essentiel : le bien être de la personne âgée. Le niveau d'exigence sur les axes jugés primordiaux est plus fort. En revanche, sur des postes où la qualité du travail impacte moins directement le quotidien de la personne âgée, la direction fait preuve de souplesse et de tolérance prenant ainsi une responsabilité sociale d'employeur » (citation de PSP : maison de retraite, 400 salariés dont $85 \%$ de femmes, $5 \%$ de bénévoles).

Dans les réponses qui suivent, un impact spécifique inhérent aux valeurs ESS est perceptible dans plusieurs registres de la GRH.

En matière de recrutement, notamment, l'association IJG (association d'hébergement de personnes âgées, 81 salariés en ETP) mentionne la «recherche d'une honnêteté (réciproque) dans les échanges pour permettre la rencontre ».

Dans un esprit égalitaire et d'ouverture, l'association LP (association spécialisée dans l'accueil d'enfants en difficulté sociale : 450 salariés en ETP) exprime le propos suivant : «donner sa chance, réellement, à tous les candidats, quelle que soit leur origine ».

Une coopérative (AFO : organisme de formation continue pour les travailleurs sociaux, 4 salariés permanents + 60 intervenants formateurs vacataires) insiste également sur ce point qui rejoint la question de la diversité : «recrutement selon les compétences techniques et capacités à "vivre" les valeurs coopératives sans discrimination / sexe - âge - nationalité - handicap...».

En matière de formation, le souci de prendre en compte les individus (valeur forte en ESS) est perceptible dans la réponse suivante: «entendre les aspirations du salarié, et l'accompagner, dans son intérêt et celui de l'entreprise » (citation de LP : association spécialisée dans l'accueil d'enfants en difficulté sociale : 450 salariés en ETP).

$\mathrm{Au}$ contraire de la coopérative qui mentionnait une échelle de salaires de 1 à 7 , une autre coopérative (SO : coopérative d'activité et d'emploi, 28 ETP pour un effectif total de 76 personnes) déclare une grille de salaires avec un écart maximum de $30 \%$ entre les salaires les plus et les moins élevés, ce qui renvoie à l'objectif de réduction des inégalités évoqué plus haut comme l'une des valeurs caractéristiques de l'ESS.

traditionnelles sont souvent vouées à l'échec; en témoignent les réticences des dirigeants de l'économie sociale à utiliser les termes de management » (Davister, op. cit., p. 17). 
Le principe démocratique transparaît dans la citation ci-dessous relative à l'organisation du travail: «nos changements d'organisation se font à partir d'une analyse collective et participative, et chacun des changements fait l'objet d'une communication anticipée au personnel (jusqu'à 3 mois) » (citation de HI, mutuelle de retraite, 77 personnes dont $80 \%$ de femmes).

On retrouve le même principe participatif appliqué à la gestion des compétences : «Tous les salariés disposent d'une définition de fonction inspirée des référentiels métiers à laquelle ils ont participé » (citation de CAP : association d'accueil d'adultes handicapés, 100 salariés et 20 bénévoles).

Le souci de trouver un équilibre entre la charge de travail et une certaine forme de respect des individus émerge dans les citations suivantes relatives à l'organisation du travail : «préserver les équilibres vie personnelle / vie professionnelle, sans oublier la priorité qui est la réponse aux usagers: cependant le confort des personnels permet de donner du confort aux résidents » (citation de IJG : association d'hébergement de personnes âgées, 81 salariés en ETP) ; «l'un de nos mots d'ordre dans l'équipe d'encadrement est : prendre soin de nos résidants implique de prendre soin de nos salariés! Ce postulat nous aide bien aussi dans nos Négociations Annuelles Obligatoires, ainsi que dans le CHSCT...» (citation de RB : maison d'accueil spécialisée pour personnes handicapées, 55 salariés en ETP).

A l'inverse d'une association qui rejette tout lien entre les valeurs de l'ESS et la question sensible de la rupture du contrat de travail, l'un des répondant (mutuelle) exprime le souci d'éviter une forme d'éviction brutale des individus : «nos procédures disciplinaires (outre le fait de respecter strictement la loi) sont construites en essayant de mettre tout en æuvre pour éviter le licenciement (on peut prévoir un accompagnement de la personne, par exemple)» (citation de HI : mutuelle de retraite, 77 personnes dont $80 \%$ de femmes). Ce point rejoint les valeurs de l'ESS. C. Davister (op. cit. p. 32) considère également qu'un licenciement est vécu comme un échec en ESS. Et ce, de manière plus forte encore, étant donné l'importance de la dimension affective dans la relation de travail.

La moitié des répondants a mentionné des difficultés de recrutement. C'est particulièrement le cas des structures actives dans le domaine médico-social (maison de retraite, hébergement de personnes handicapées, hébergement d'enfants en difficultés sociales) qui ont besoin de compétences relativement spécifiques (orthophonistes, médecin psychiatre, enseignants pour 
déficients auditifs, éducateurs, infirmiers, etc.), même si les compétences en question ne sont pas forcément très élevées en termes de qualification (plusieurs maisons de retraite rencontrent des difficultés pour recruter et remplacer des aides-soignantes) ${ }^{1}$.

En réponse à ces difficultés de recrutement, justifiées entre autre par des conditions de travail difficiles, plusieurs structures recourent à des contrats de travail précaires. Ce qu'elles semblent déplorer comme le montrent les propos ci-dessous, à l'inverse des entreprises classiques qui apprécient de trouver notamment dans l'intérim et les CDD une solution pratique aux surcroîts d'activité et un moyen de mettre à l'épreuve et donc de tester des futures recrues (en CDI) possibles (Everaere, 1999 ; Abadie et al., 2008).

« Recours aux CDD trop important pour la qualité de travail et la vie des équipes. Recours trop important à l'intérim du fait de la pénurie de personnel soignant depuis octobre 2007 » (Citation de IJG : association d'hébergement de personnes âgées, 81 salariés en ETP)

«La précarité des financements publics, leur incertitude nous amène à faire des CDD, à embaucher à bas prix, à ne pas pouvoir fidéliser les gens »(Citation de AA : association d'accueil de personnes alcooliques et en situation d'addiction, 7 salariés en ETP +3 bénévoles)

«Il nous arrive d'avoir recours temporairement (et en urgence !!!) à l'intérim pour palier aux absences de nos infirmières » (Citation de RB : maison d'accueil spécialisée pour personnes handicapées, 55 salariés en ETP)

Cette perception contrastée (plutôt subie pour les structures de l'ESS, plutôt choisie et appréciée pour les entreprises classiques ${ }^{2}$ ) en matière de recours à des contrats de travail précaires, constitue une ligne de démarcation entre les entreprises de l'ESS et celles de l'économie classique. La question des valeurs et la place prioritaire de l'humain dans le projet, caractéristiques de l'ESS, peut expliquer ce phénomène.

\footnotetext{
${ }^{1}$ Ce phénomène de pénurie de ressources humaines est bien connu du secteur médico-social dans son ensemble : «Dans le domaine du tertiaire, les tensions entre offres et demandes d'emploi diminuent pour tous les métiers, sauf pour certains métiers de la santé, action sociale, culturelle et sportive comme les aides soignantes et les infirmiers qui voient progresser vivement le ratio des tensions » (Tabet et Viney, 2008).

2 «Pour la moitié des employeurs interrogés, l'intérim et le CDD constituent une pratique d'embauche courante (...) $53 \%$ d'entre eux y ont recours pour constituer une « super période d'essai » (...) « Ca permet de garder les bons » (Abadie et al., op. cit., pp. 3,6) .
} 
Nous restituons une dernière citation qui a été exprimée en guise de réponse à cette question de l'impact des valeurs de l'ESS sur les pratiques de GRH. Citation qui émane de la même personne qui évoquait plus haut sa double expérience des entreprises classiques et des entreprises de l'ESS :

«Je n'ai pas changé de valeurs en changeant de secteur d'activité. Aussi, je ne suis pas certain d'avoir des buts différents en matière de GRH dans ce secteur plutôt que dans un autre.

Question: les valeurs de l'ESS servent probablement les usagers des établissements et services. En revanche, servent-elles toujours le personnel? Ne serait-ce pas plutôt des valeurs individuelles qui impacteraient davantage la GRH? Ces valeurs individuelles n'étant pas forcément tout à fait les mêmes que les valeurs de l'ESS : on trouve autant de comportements racistes, de jalousies, d'enjeux de pouvoir dans le secteur de l'ESS que dans le secteur industriel ! ( (citation de CBF : responsable d'un centre de santé, 45 salariés âgés de 48 ans en moyenne).

Pour résumer, les valeurs énoncées plus haut de façon relativement consensuelle (dans les réponses à la question 3) trouvent des applications plus diversifiée et nuancée lorsqu'elles sont déclinées dans des pratiques concrètes de GRH ${ }^{1}$. Certaines structures restent concentrées sur la spécificité de leurs ressources (personnes handicapées, volontaires) pour justifier des pratiques de GRH particulières. Les autres se partagent entre des pratiques que nous qualifions de classiques, et des pratiques imprégnées d'une certaine sensibilité à l'humain.

\section{En guise de conclusion}

Cette recherche exploratoire s'intéresse à un secteur relativement peu étudié, car à la fois relativement minoritaire en termes d'effectifs (10\% de l'emploi), et surtout difficile à cerner en raison de sa grande hétérogénéité.

L'une des limites importantes à ce travail réside dans la taille réduite de l'échantillon étudié, qui interdit la moindre extrapolation statistique. Une autre limite importante à ce travail est que nous ne disposons que de déclarations (les réponses formulées par écrit dans les retours

\footnotetext{
${ }^{1}$ P. Valeau (2003, p. 20) a également constaté, au au-delà des discours ou des déclarations d'intention, de grandes différences dans les pratiques de GRH au sein, en particulier, des associations : « Les associations nous ont donné à voir des gestions de ressources humaines très différentes les unes des autres. Si un grand nombre semble effectivement fonctionner à base d'implication morale, certaines prennent des formes relativement
} 
de mail). Or, nous n'avons pas corroboré ces déclarations par des entretiens en tête à tête notamment auprès des collaborateurs des structures en question, ni par des observations de terrain in situ.

Cependant, cette recherche tente de mettre en évidence les liens particuliers entre les valeurs supposées « humaines » attribuées à l'ESS et des pratiques de management de ces ressources humaines. Si nombre de pratiques et de problématiques exprimées ici apparaissent banales au regard des discours classiques en matière de GRH, nous avons pu repérer à plusieurs reprises des liens entre les valeurs «humaines » de l'ESS et les pratiques de GRH. Les principaux liens sont repris et résumés ci-dessous :

- $\quad$ une certaine réticence à recourir à des contrats de travail précaires.

- des écarts de rémunération (parfois) réduits, (une coopérative mentionne cependant des écarts de rémunération de 1 à 7).

- $\quad$ une certaine indulgence dans les recrutements (« donner sa chance à tous les candidats, quelle que soit leur origine »),

- la revendication d'un fonctionnement démocratique, transparent, et d'une propriété collective de l'outil de production (pour les coopératives en particulier),

- l'importance d'un ancrage fort dans un territoire donné associé à une connaissance proche (importance du principe de proximité ${ }^{1}$ ), personnalisée (chaque personne est différente ou singulière) et durable des acteurs de ce territoire ;

- $\quad$ le souci d'un équilibre vie professionnelle / vie personnelle des salariés ${ }^{2}$;

- l'octroi d'une certaine liberté dans la gestion du temps de travail, dans le respect des contraintes collectives.

Sous forme de boutade, et probablement pour exprimer l'impossibilité d'affirmer une gestion complètement alternative des ressources humaines en ESS, l'un de nos interlocuteurs a suggéré d'inverser l'ordre des lettres du sigle GRH, en proposant le sigle GHR : gestion humaine des ressources, pour caractériser les principes de fonctionnement des entreprises de l'ESS.

autoritaires, d'autres encore se fondent sur des rapports essentiellement professionnels, quelques unes, à l'image du Progrès, admettent un véritable échange »

${ }^{1}$ Il est intéressant de souligner que ce principe de proximité est à la fois propre au TPE (Torrès et Delmar, 2006), et aux valeurs humaines de l'ESS.

${ }^{2}$ Sur ce point en particulier, il importe de préciser que les structures de l'ESS n'ont pas le monopole de cette préoccupation. Des entreprises classiques telles que Boiron, par exemple, expriment également ce souci de concilier travail et hors travail pour leurs salariés (Fairise, 2003). 


\section{Bibliographie}

Abadie R., Fontanet ML., Baudrier O., Charvet A., (2008), «CDD, intérim : quelles bonnes pratiques pour allier performance de l'entreprise et meilleures conditions d'emploi », DRTEFP Rhône-Alpes, DDTEFP de l'Ain, ARAVIS, décembre 2008.

Archambaud E., (1996), Le secteur sans but lucratif, Paris, Economica.

Berry JB., Dubois H., (2008), «L'emploi dans les très petites entreprises en juin 2007», Premières informations et premières synthèses, DARES, $\mathrm{n}^{\circ} 51.5$, décembre.

Clergeau C., Dussuet A., (2004), «Les entreprises sociales en quête de professionnalisation », Revue des Etudes Coopératives, Mutualistes et Associatives, n²94, novembre 2004.

CNESS (2008), Chambre Nationale de l'Economie Sociale et Solidaire, « Panarama de l'ESS en France et dans les régions », données au 31/12/2006, source INSEE-CLAP, décembre.

Colletis G., Gianfaldoni P., Richez-Battesti N., (2004), «Economie sociale et solidaire, territoires et proximité », Revue des Etudes Coopératives, Mutualistes et Associatives, n² 296, mai.

Davister, C., (2006), «La gestion des ressources humaines en économie sociale », Les Cahiers de la Chaire Cera, vol. $\mathrm{n}^{\circ} 1$, mai.

Demoustier D., (2006), « Débats autour de la notion d'économie sociale en Europe », Revue des Etudes Coopératives, Mutualistes et Associatives, ${ }^{\circ} 300$, mai

Diloisy C., Dupé A. (2007), «L'ESS rassemble près de $10 \%$ des salariés de la région », Lettre de L'INSEE Rhône-Alpes, n 76, juin 2007.

Draperi J.F., (2003), «L'économie sociale et solidaire : utopie, alternative, réforme ? », Revue des Etudes Coopératives, Mutualistes et Associatives, ${ }^{\circ}$ 290, novembre.

Everaere C., (1999) "Les effets pervers de la flexibilité quantitative », Revue Française de Gestion, ${ }^{\circ} 124$, juin-juillet-août.

Fairise A., (2003), «Christian Boiron n'instille pas le social à dose homéopathiques », Liaisons Sociales, septembre.

INSEE (2003), «L'économie sociale et solidaire : associations, mutuelles et coopératives en Rhône-Alpes », Les dossiers de l'INSEE, n 146, mai 2003.

Jeantet, T. (2006) Economie sociale, la solidarité au défi de l'efficacité, Les études de la Documentation Française.

Mayaux F., (2001), «La mobilité professionnelle des cadres de l'économie marchande vers l'économie sociale : pourquoi ? comment?», Revue des Etudes Coopératives, Mutualistes et Associatives, $\mathrm{n}^{\circ} 281$, juillet 2001. 
Persais E., (2006), «Bilan sociétal : la mise en œuvre d'un processus RSE au sein du secteur de l'économie sociale », Revue des Etudes Coopératives, Mutualistes et Associatives, $\mathrm{n}^{\circ} 302$, novembre.

Tabet N., Viney X., (2008) «Les tensions sur le marché du travail au premier trimestre 2008 », Premières informations et premières synthèses, DARES, n 30.2, juillet 2008.

Tchernonog V., (2008), «Les grandes évolutions su secteur associatif français », Revue des Etudes Coopératives, Mutualistes et Associatives, juillet 2008.

Torrès O., Delmar F. (2006), "Smallness of firm and HRM : the key role played by proximity », 20 $0^{\text {th }}$ Conference of European Council of Small Business (ECSB), Rent XX, Bruxelles, Belgique.

Ulrich V., Zilbermann S., (2007), «De plus en plus d'emplois à temps partiel au cours des vingt cinq dernières années $»$, Premières informations et premières synthèses, DARES, $\mathrm{n}^{\circ}$ 39.3, septembre 2007.

Valéau P., (2003) «Différentes manières de gérer les associations », Revue Française de Gestion, vol. 29, $\mathrm{n}^{\circ}$ 146, septembre octobre. 